Macedonian Pharmaceutical Bulletin, 66 (Suppl 1) 227 - 228 (2020)

Online ISSN 1857 - 8969

UDC: $615.015 .14: 620.3$

DOI: 10.33320/maced.pharm.bull.2020.66.03.113

Short communication

\title{
Effects of formulation and sol-gel synthesis conditions on physical stability and chemical structure of organomodified silica nanoparticles: a screening study
}

\author{
Teodora Dimkovska ${ }^{1}$, Beti Djurdjic ${ }^{2}$, Katerina Goracinova $^{3}$, Boban Mugosa ${ }^{4}$, \\ Nikola Geskovski ${ }^{1}$ * \\ ${ }^{1}$ Institute of Pharmaceutical technology, Faculty of Pharmacy, Ss. Cyril and Methodius University, \\ Majka Tereza 47, 1000, Skopje, N. Macedonia \\ ${ }^{2}$ Department of Pharmacy, Faculty of Medicine, University of Montenegro, Kruševac bb, \\ 81000 Podgorica, Montenegro \\ ${ }^{3}$ College of Pharmacy, Qatar University, PO Box 2713, Doha, Qatar \\ ${ }^{4}$ Institute of Public Health, Džona Džeksona bb, 81000 Podgorica, Montenegro
}

\section{Introduction}

Nanoparticles (NPs) have drawn increasing interest from every branch of medicine as potential carriers for targeted drug delivery in the optimum dosage range providing increased therapeutic efficiency, and at the same time minimizing the side effects. This is especially important in cancer chemotherapy, where patients experience severe side effects that seriously affect the quality of life. However, many factors (i.e. particle size, zeta potential, drug loading, stability, interactions with the biological milieu) influence the overall efficacy of the nanoparticulated drug carriers. Therefore, an in-depth approach is needed to design such formulation, evaluate and understand its biological behavior and predict its possible toxicity and therapeutical effects.

The aim of our study was to screen the effects of preparation procedure and formulation factors $(\mathrm{pH}$, silica precursors ratio) on the physical stability (particle size, polydispersity index, zeta potential) in different relevant media, and the chemical structure of silica and organomodified silica nanoparticles.

\section{Materials and Methods}

Materials

Tetraethoxysilane (TEOS) and 3-aminopropyl triethoxysilane (APTES) were purchased from Sigma Aldrich (Germany); Ethanol (96\%), Sodium hydroxide and Acetic acid were purchased from Alkaloid AD (N. Macedonia). All other reagents and chemicals used were of analytical grade.

\section{Methods}

Previously described sol-gel method was used for the preparation of the silicate nanoparticles (Djurdjic et al., 2018). The process involves hydrolysis and condensation of metal alkoxides $\left(\mathrm{Si}(\mathrm{OR})_{4}\right)$ such as TEOS, APTES in the presence of mineral acid (e.g., $\mathrm{HCl}$ ) or base (e.g., $\mathrm{NH}_{3}$ ) as a catalyst. Pure TEOS and TEOS/APTES NPs in the ratio of 99:1 were prepared. The physical stability of formulations was tested by diluting the corresponding sample volume of $300 \mu \mathrm{g}$ NPs with

\footnotetext{
*ngeskovski@ff.ukim.edu.mk
} 
an appropriate medium $(0.1 \mathrm{M} \mathrm{HCl}$, phosphate buffer 4.5 and 7.4) of up to $3 \mathrm{~mL}$ and incubating at $37{ }^{\circ} \mathrm{C}$. Appropriate volume $(100 \mu \mathrm{L})$ was taken 1, 3, 5 and 7 hours after the start of incubation. Particle size (PS), polydispersity index (PDI) and zeta potential (ZP) analysis were performed by dynamic light scattering using the Malvern Zetasizer Nano ZS90 (Malvern Panalytical, UK). Fourier transform infrared spectra were acquired on freeze-dried samples using Nicolet iS10 (Thermo Scientific, USA). Multivariate analysis (SIMCA 14, Umetrics, Sweden) was employed to investigate the possible effects of the formulation and process variables upon the NP chemical structure (FTIR spectra) and in vitro stability of their PS, PDI and ZP.

\section{Results and Discussion}

Categorical PCA X\&Y models were employed to identify the effects of the independent variables ( $\mathrm{pH}$ during the sol-gel synthesis of NP, silica precursors TEOS/TEOS-APTES) upon the NP's PS, PDI, ZP stability trends and FTIR spectra. Each model contained two main components explaining $\left(\mathrm{R}^{2} \mathrm{X}\right) 90.8,98.3,99.4$ and $98.8 \%$ of the variations in PS, PDI, ZP and FTIR spectra, respectively. The predictivity coefficient $\left(\mathrm{Q}^{2}\right)$ was within satisfactory limits $(0.825-0.986)$ for all models. The results revealed that the hydrodynamic diameter of the particles and their stability in $\mathrm{pH} 7.4$ was mainly governed by the silica precursors (TEOS/TEOSAPTES) employed in the formulations, while no significant variations among the formulations in PS stability were observed in $\mathrm{pH} 1$ and 4.5 . The PDI of the formulations in all tested media was affected by both the preparation procedure and silica precursors. The stability of $\mathrm{ZP}$ at $\mathrm{pH} 1$ was mainly influenced by the silica precursors, while the $\mathrm{pH}$ of the preparation procedure mainly affected the $\mathrm{ZP}$ stability at $\mathrm{pH}$ 7.4. All observed effects were probably due to the surface-oriented amino groups that govern the surface potential of the particles affecting particle growth during synthesis and stability during incubation, and the $\mathrm{pH}$ of the sol-gel synthesis process which also affects the silica hydrolysis rate and condensation behavior, thus resulting in noticeable effects on the PS, PDI and ZP (Wu et al., 2013). The SNV derived FTIR data revealed that the position and intensity of the bands at 1549, 1410, 1090, 902 and $770 \mathrm{~cm}^{-1}$ were most affected by the silica precursors used in the synthesis procedure, while the $\mathrm{pH}$ of the sol-gel synthesis procedure didn't demonstrate any significant effect on the FTIR spectra. The bands at 1090 and $770 \mathrm{~cm}^{-}$ ${ }^{1}$ correspond to the antisymmetric and symmetric stretching of the Si-O-Si species from the silica NP matrix and were blue-shifted in the pure $\mathrm{SiO}_{2}$ matrices. The bands at 1549 and $1410 \mathrm{~cm}^{-1}$ originate from the deformation $\mathrm{CH} 2$ and $\mathrm{NH} 2$ vibrations of the alkylamino chain, which appeared only in the APTES based formulations, while the band at 902 $\mathrm{cm}^{-1}$ is associated with the $\mathrm{Si}-\mathrm{OH}$ and $\mathrm{Si}-\mathrm{O}-\mathrm{R}$ stretching modes (Brinker and Scherer, 2013).

\section{Conclusion}

Silica nanoparticles can be prepared by the hydrolysis reaction of TEOS and APTES in ethanol using mineral acid or base as a catalyst using the solgel method. In this screening study, using multivariate analysis, we have observed the effects of the silica precursors ratio and $\mathrm{pH}$ of the sol-gel synthesis procedure upon the stability patterns of the formulations (PS, PDI and ZP) in different biorelevant media and their surface chemical structure. The generated data pool could be used as a platform for further development and optimization of drug-loaded silica-based nanoparticles.

\section{Acknowledgements}

The authors would like to acknowledge the support of Ministry of Education and Science of N. Macedonia, Ministry of Science of Montenegro and the CEEPUS CEKA PharmTech Network (CIII-RS1113-03-1920).

\section{References}

Brinker, P.J., Scherer, G.W., 1990. Sol-gel science: The physics and chemistry of the sol-gel processing. Academic Press, San Diego, CA, USA.

Djurdjic, B., Goracinova, K., Geskovski, N., Mugosa, B., 2018. Amino-modified silica nanoparticles as carriers for 5-fluorouracil: influence of preparation process parameters on physico-chemical properties and drug release. Arh. Farm. 68, 620.

Wu, S.H., Mou, C.J., Lin, H.P., 2013. Synthesis of mesoporous silica nanoparticles. Chem. Soc. Rev. 42, 3862-3875.

Maced. Pharm. Bull. 66 (Suppl 1) 227 - 228 (2020) 